\title{
On spatial variations of magnetic field and superthermal electron distribution in cm-radio burst source
}

\author{
Leonid V. Yasnov ${ }^{1}$ and Marian Karlický ${ }^{2}$ \\ ${ }^{1}$ Radiophysical Research Institute, St.-Petersburg State University \\ Ul'yanovskaya 1, St.-Petersburg, 198504, Russia \\ ${ }^{2}$ Astronomical Institute, Academy of Sciences of the Czech Republic \\ 25165 Ondrejov, Czech Republic
}

\begin{abstract}
The paper presents a new method of an estimation of spatial variations of the magnetic field and superthermal electron distribution in solar cm-radio burst sources. The method is based on the analysis of several burst spectra recorded in the different moments of time and on the minimization of the difference between the theoretical and observed radio fluxes. It is found that the measure of the spatial variations of superthermal electron distribution in the radio source is always greater than that for the magnetic field. In most cases this measure has a minimum at the impulsive phase of cm-radio bursts.
\end{abstract}

Keywords. Sun: radio radiation Methods: data analysis

Many papers have been devoted to a study and interpretation of spectral characteristics of the centimeter radio bursts. These characteristics can be used for a diagnostics of the cm-radio burst sources. An attempt to estimate some physical parameters in the radio source from radio burst spectra has been presented by Böhme et al. 1977. The authors concluded that their inversion procedure is ambiguous.

For the X-ray bremsstrahlung and synchrotron emission various inversion methods have been described in the literature (Brown et al. 1983; Brown et al. 2006; Kontar et al. 2004; Prato et al. 2006).

In the present paper, for an analysis of the cm-burst radio spectra, a new inversion method is suggested. We propose to reduce an ambiguity of the problem by comparing the radio spectra taken at different times during one specific cm-radio burst.

Let us assume that the radio source has a form of a magnetic loop segment with the squared cross section. The length of this segment along the magnetic field direction (the $l$ coordinate) is $L . \Delta_{0}$ is the length of the side of the square section at the segment base as well as a location of the segment base at the $l$-coordinate. Then, for the square side $\Delta$ and the magnetic field $B$ in the radio source we can write: $\Delta=\Delta_{o}\left(\frac{l}{\Delta_{o}}\right)^{-\mu}$ and $B=B_{o}\left(\frac{l}{\Delta_{o}}\right)^{2 \mu}$, where $B_{o}$ is the magnetic field at the segment base. Furthermore, let us assume that in the radio source the superthermal electrons are distributed as follows: $n=n_{o}\left(\frac{l}{\Delta_{o}}\right)^{-\nu}$, where $n_{o}$ is the density of the superthermal electrons at the segment base. The parameters $L, \Delta_{o}, B_{o}, n_{o}, \mu$, and $\nu$ are unknown parameters and they need to be determined, see the following.

In the centimeter wavelength range the emission is usually produced by the gyrosynchrotron emission mechanism. Therefore all the following analysis is based on this mechanism. In agreement with Dulk (1985), Bastian et al. 1998, Nindos et al. 2000 we consider the isotropic distribution functions of electrons in the source and thus we can use the emission $\eta$ and absorption $k$ coefficients derived by Dulk \& Marsh (1982). 
We suppose that $L \gg \Delta_{o}$. Because most of the cm-burst sources are located at the top of flare loops (White et al. 2002, Minoshima et al. 2008) and thus the angle between the line of sight and the magnetic field vector $\theta$ is much greater than zero, the following integration is made only along the axis of the source (in such approximation the integration along the line of sight is not necessary). Then using the emission and absorption coefficients from the paper by Dulk \& Marsh (1982), the radio flux measured in SFU at the specific frequency can be written as

$$
\begin{aligned}
F(f) & =\frac{1}{10^{-19} R^{2}} \int_{\Delta_{o}}^{L} \frac{\left(1-e^{-k \Delta(l) / \sin (\theta)}\right) \eta \Delta(l)}{k \sin (\theta)} d l \\
& =\frac{C(f)}{10^{-19} R^{2}}\left[-n c a E_{1-n c a}(L d n A(f)) L d n^{n c a}-L d n^{n c a}+n c a E_{1-n c a}(A(f))+1\right]
\end{aligned}
$$

where

$$
\begin{aligned}
& A(f)=1.28 \times 10^{-10} \frac{n_{o} \Delta_{o}}{B_{o}} e^{-2.308 \delta} \sin (\theta)^{0.72 \delta-1.09}\left(\frac{f}{f_{B, o}}\right)^{-0.98 \delta-1.30} \equiv A o f^{-0.98 \delta-1.30}, \\
& C(f)=2.42 \times 10^{-13} \frac{B_{o}^{2} \Delta_{o}^{2}}{n c-1} e^{-0.544 \delta} \sin (\theta)^{0.07 \delta-1.34}\left(\frac{f}{f_{B, o}}\right)^{0.08 \delta+2.52} \equiv C_{o f}^{0.08 \delta+2.52},
\end{aligned}
$$

and $f$ is the emission frequency, $\delta$ is the energy spectral index of superthermal electrons, $E_{n}(x)$ is the integral-power function, $n c a=\frac{1-n c}{n a}, L d n=L d^{n a}, n c=(0.16 \delta+2.04) \mu$, $n a=(1.96 \delta-0.4) \mu-\nu, L d=\frac{L}{\Delta_{o}}, f_{B, o}=\frac{e B_{o}}{2 \pi m_{e} c}, R=1.49 \times 10^{13} \mathrm{~cm}$ is the Sun-Earth distance.

Considering now some real radio observations we need to find the parameters of the theoretical model which give the radio fluxes fitting the observed ones by the best way. For this optimalization procedure, we define the difference of the radio fluxes in the form

$$
\sum_{i=1}^{N}\left(\lg F\left(f_{i}\right)-\lg F^{o}\left(f_{i}\right)\right)^{2},
$$

where $F$ and $F^{o}$ are the theoretical and observed radio fluxes at one specific instant during the radio burst and at the frequency $f_{i} ; N$ is the number of considered frequencies. To find the optimal parameters of the theoretical model, we need to find the minimum of this difference for the auxiliary parameters $C_{0}, A_{0} . \delta, n c a$, and $L d n$. To reduce an ambiguity of the present task, this minimization has be done for at least two radio spectra observed at two different times during one selected radio burst. As explained in the following more spectra represent more information and better results.

There are many papers showing that changes of the magnetic field at the photospheric level during solar flares are negligible (see e.g. Rust 1972). Let us assume the same for the studied radio source (in the cm-range is usually out of the primary energy release) and later we will check the validity of this assumption. Thus the parameters $\mu, L$, and $\Delta_{0}$ will be taken constant during the whole evolution of the radio burst. Now considering two radio spectra in two different times during the radio burst, i.e. for two sets of the auxiliary parameters $\left(C_{0}, A_{0} . \delta, n c a\right.$, and $\left.L d n\right)$, which were obtained by the minimization of the foregoing relation, we can express the parameters in the radio source as follows:

$$
\begin{gathered}
\mu=\frac{25\left(n c a_{i} \ln \left(L d n_{i}\right)-n c a_{k} \ln \left(L d n_{k}\right)\right)}{n c a_{i}\left(4 \delta_{k}+51\right) \ln \left(L d n_{i}\right)-n c a_{k}\left(4 \delta_{i}+51\right) \ln \left(L d n_{k}\right)} \\
\nu_{i}=\frac{\left(4 \delta_{i}-4 \delta_{k}+n c a_{i}\left(49 \delta_{i}-10\right)\right) \ln \left(L d n_{i}\right)+n c a_{k}\left(10-49 \delta_{i}\right) \ln \left(L d n_{k}\right)}{n c a_{i}\left(4 \delta_{k}+51\right) \ln \left(L d n_{i}\right)-n c a_{k}\left(4 \delta_{i}+51\right) \ln \left(L d n_{k}\right)}
\end{gathered}
$$


Table 1. The parameters of the cm-radio burst of December 24, 1991.

\begin{tabular}{lcc}
\hline Time(UT) & $\delta$ & $\nu$ \\
\hline $18: 34: 00$ & 4.44 & $3.00 \pm 0.05$ \\
$18: 38: 02$ & 2.47 & $1.81 \pm 0.08$ \\
$18: 38: 14$ & 3.71 & $2.61 \pm 0.04$ \\
$18: 39: 26$ & 3.71 & $2.42 \pm 0.17$ \\
\hline
\end{tabular}

These relations were derived by algebraic operations of two sets of the auxiliary parameters corresponding to two radio spectra (for their designation we use the indexes $i$ ). Let's note that definition of parameters $\mu, \nu$ does not demand knowledge of the angle $\theta$. The numerical experiments shows that for small amount of frequencies only the parameters $\mu, \delta$, and $\nu$ are stable (within $15 \%$ of the original values); other parameters differ sometimes several orders of the magnitude.

Let us use the radio spectra which were observed at 18:33 - 18:40 UT, 24 December 1991, at the Sagamore Hill Station, and were analyzed in detail in the paper by Willson (1993). The resulting parameters $\delta$ and $\nu$ obtained for this burst can be seen in Table 1 . Because values of $\nu$ are calculated for different pairs of the spectra, and because in each time instant three values of $\nu$ can be determined, then an accuracy of the parameter can be estimated. The number of combinations of the spectral pairs is 6 and in all such cases a value of the parameter $\mu$ was determined. It gave us the possibility to estimate an accuracy of determination of this parameter as well as estimate the accuracy of the above made assumption about the constant value of the parameter $\mu$ during the radio burst. As a result we have $\mu=0.39 \pm 0.04$, i.e. an inaccuracy is small, which confirms the used assumption and the quality of the determination of these parameters by the proposed procedure.

The impulsive phase of the radio burst takes place at 18:38:02 UT. It can be seen that in the impulsive phase the values of $\delta$ and $\nu$ sharply decrease. This effect can be connected with an increase of the effectiveness of the acceleration of electrons to higher energies at times of the impulsive burst phase, which corresponds to a decrease of $\delta$ and to an increase of the emission volume in this burst phase. Thus, the results are in good agreement with the physics of the burst, and it one again confirms effectiveness of the proposed procedure.

To confirm the found evolution of the parameter $\nu$ during the December 24, 1991 radio burst, we used a set of the spectral observations published in Solar Geophysical Data Journal. We used data for bursts recorded on 11 Jan. 68, 14 Feb. 68, 03 Apr. 68, 09 Jul. 68, 21 Aug. 68, 09 Feb. 69, 27 Mar. 69, 17 May 69, 20 May 70, 14 Jul. 70, 12 Aug. 70, 24 Apr. 71 and 07 Aug. 72. From spectral point of view these data are not so good as those for the December 24, 1991 event. However, their number is sufficient for some statistical analysis and thus appropriate to make a statistical verification of this phenomenon. We take into the set of events only the radio bursts with the flux maxima at high-frequencies, i.e. the radio bursts for which reliable parameters $\delta$ can be determined. By this way we have selected 68 radio spectra for 13 radio events.

Inaccuracies of observations, minimization procedures and deviations from used assumptions result in the error range of the computed parameters $\mu$ and $\nu_{i}$ for all instants during the radio bursts. The error range was estimated from all values of $\mu$ and $\nu_{i}$ which were determined for all combinations of spectral pairs for specific instants during the radio bursts. 
In this process of the parameter determination, it is possible to verify the validity of the assumption about the constant value of the magnetic field strength during radio bursts. For the radio bursts observed in August 12, 1970 and August 21, 1968 this condition breaks (the value of the relative inaccuracy of $\mu$ and $\nu$ is large (about 50\%), in other cases the relative inaccuracy was about 10\%), i.e. the magnetic field structure in the radio source changes during the radio burst. It can be caused by spatial displacements of the sources of the elementary bursts. For other 11 bursts we can say that their magnetic structure is stable. It means that their changes influence the determination of the parameter $\nu$ by a negligible way.

Almost all events show a decrease of the parameter $\nu$ at the beginning phase of the radio burst. Some exceptions are connected with the following. As we mentioned above, values of $\nu$ for the August 12, 1970 event are not trustworthy. For the July 14, 1970 event all data were observed after the maximum burst phase. Only the February 9, 1969 event represents a real exception.

Summarizing the results of all tests and data analysis we can conclude as follows:

1. For all analyzed radio bursts the measure of the spatial variations of the superthermal electron distribution $(\nu=0.7-4.2)$ is greater than that for the spatial variations of the magnetic field $(\mu=0.4-0.5)$.

2. Almost all events indicate a decrease of the parameter $\nu$ at the beginning phase of the radio burst.

\section{Acknowledgements}

This research was supported by Grant 06-02-16502 of the RFBR and by Grant IAA300030701 of the Grant Agency of the Academy of Sciences of the Czech Republic.

\section{References}

Bastian, T. S., Benz, A. O., \& Gary, D. E. 1998, ARAA, 36, 131.

Böhme, A., Fürstenberg, F., Hildebrandt, J., Saal, O., Krüger, A., Hoyng, P., \& Stevens, G. A. 1977, Solar Phys., 53, 139

Brown, J. C., Craig, I. J. D., \& Melrose, D. B. 1983, Ap\&SSS, 92, 105

Brown, J. C., Emslie, A. G., Holman, G. D., Johns-Krull, C. M., Kontar, E. P., Lin, R. P., Massone, A. M., \& Piana, M. 2006, ApJ, 643, 523

Dulk, G. A. $1985, A R A A, 23,169$

Dulk, G. A. \& Marsh, K. A. 1982, ApJ, 259, 350

Kontar, E. P., Piana, M., Massone, A. M., Emslie, A. G., \& Brown, J. C. 2004, Solar Phys., 225,293

Minoshima, T., Yokoyama, T., \& Mitani, N. 2008, ApJ, 673, 598

Nindos, A., White, S. M., Kundu, M. R., \& Gary, D. E. 2000, ApJ, 533, 1053

Prato, M., Piana, M., Brown, J. C., Emslie, A. G., Kontar, E. P., \& Massone, A. M. 2006, Solar Phys., 237, 61

Rust, D. M. 1972, Solar Phys., 25, 141

Solar Geophysical Data 1968-1972, NOAA, Boulder, USA

White, S. M., Kundu, M. R., Garaimov, V. I., Yokoyama, T., \& Sato, J. 2002, ApJ, 576, 505

Wilson, R. F. 1993, ApJ, 413, 798 